\title{
MoyaMoya Disease in a Child: a Case Report
}

\author{
Joarder MA ${ }^{1}$, Chandy $\mathrm{M}^{2}$
}

\begin{abstract}
Acute stroke is an infrequent disease of pediatric age group patients. MoyaMoya is a rare cerebro vascular disease of unknown etiology. We report a case of 14-year-old male child who presented with right-sided hemiplegia and diagnosed as a MoyaMoya disease. MoyaMoya disease is rare, but important as a cause of cerebral stroke in children. Cerebral revascularization surgery leads to favorable outcome.
\end{abstract}

\section{Introduction}

MoyaMoya disease is a rare cerebrovascular disorder of unknown etiology consisting of stenosis or occlusion of main cerebral arteries which causes an abnormal vascular network at the base of the brain. The Japanese term "moyamoya" (something hazy, like a puff of smoke drifting in the air) describes the angiographic appearances. The vascular abnormalities are bilateral in the disease, while unilateral appearance can occur in other conditions such as neurofibromatosis, tuberous sclerosis, tuberculus meningitis, etc. The disease commonly presents with strokes in childhood, while in adults subarachnoid and intracranial hemorrhage is the prevailing presentation. ${ }^{1}$

\section{Case history}

A 14 year-old male child was admitted at our hospital with complaints of weakness over right upper and lower limbs for 30 days. There was also history of sudden fall several times specially during playing in last 5 years.

There was no history of fever, convulsion, head injury, and ear discharge. There was no history of delayed mile stones. On examination patient was found to be conscious but disoriented. There were no neurocutaneous markers or asymmetry of face. Apart from temperature of $99^{\circ} \mathrm{F}$ other vital signs were normal. On neurological examination, there were no signs of meningeal irritation, gait was hemiplegic, tone was decreased over rightside, power was 2/5 over right upper and lower limb, deep tendon reflexes were exaggerated, and planter was extensor over right side. All other systems were found to be normal.

Hematological examination was normal. MRI brain was suggestive of MoyaMoya disease and multiple watershed zone infarcts more on left side (Fig 1). MR angiography brain revealed MoyaMoya collaterals in both basal ganglia region, which gives "puff of smoke" appearance (Fig 2).

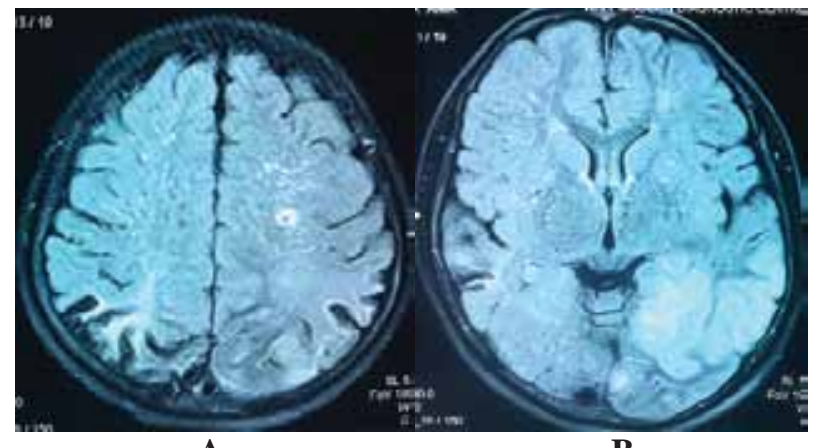

A

B

Fig 1: MRI brain FLAIR image $A$. reveals MoyaMoya vessels as a linear structure crossing the white matter (medullary streaks) B. shows MoyaMoya vessels and multiple infarct (left $>$ right). 


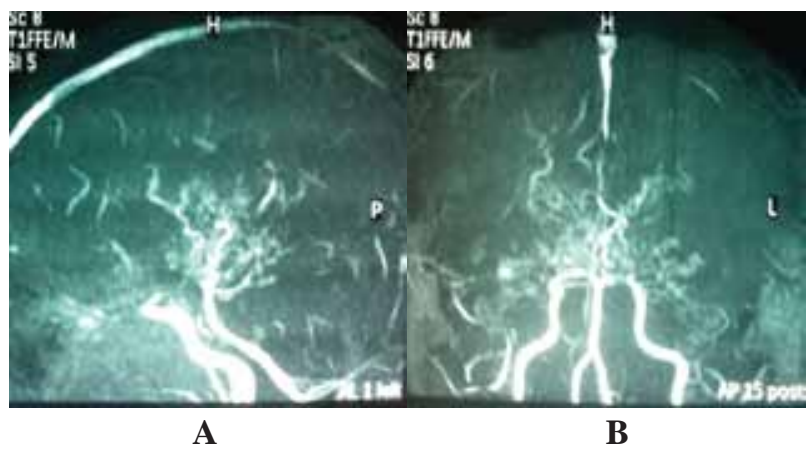

Fig 2: MR angiography shows stenosis or occlusion in the terminal ICA and posterior circulation with MoyaMoya collaterals and typical "puff of smoke" appearance (Stage 3).

We prepared the patient for superficial temporal artery to middle cerebral artery anastomosis on right side. During surgery we found arterial size $<1 \mathrm{~mm}$, so we had to do indirect bypass surgery that is encephalomyosynangiosis, a procedure that involves laying the temporalis muscle over the surface of the brain (Fig 3). Post operative period was uneventful. During discharge patient was fully conscious, oriented and right hemi paresis (motor power 2/5). Our plan is to follow up the patient, if there is significant improvement, we will do same surgery on the left side.

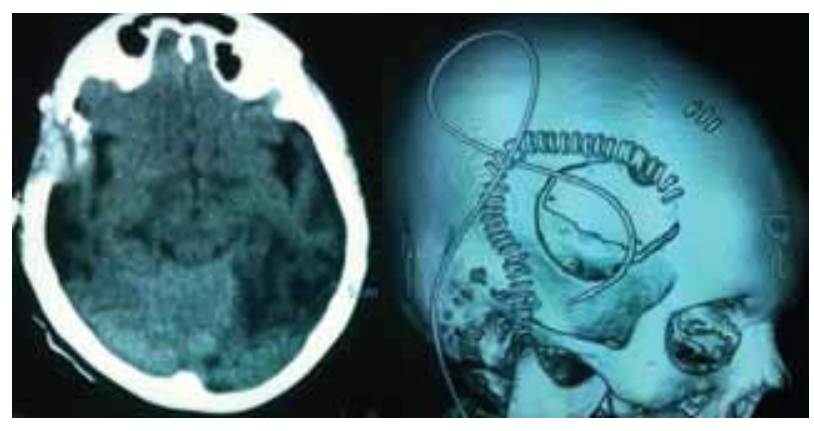

A B

Fig 3: Post operative CT brain shows vascularized temporalis muscle lies directly on the brain (A). Post operative CT brain shows 3D reconstruction of Right frontotemporoparietal craniotomy.

\section{Discussion}

Moyamoya disease was first described in Japan by Takeuchi and Shimizu in 1963. Moyamoya disease is a chronic, progressive occlusion of the circle of Willis arteries that leads to the development of characteristic collateral vessels seen on imaging, particularly cerebral angiography. The appearance of these small, multiple vessels at the base of the brain on angiography was originally described by the Japanese term moyamoya, which translates to "puff of smoke." Moyamoya disease occurs predominantly in Japanese individuals but has been found in all races with varying age distributions and clinical manifestations. As a result, moyamoya disease has been under recognized as a cause of ischemic and hemorrhagic strokes. 2,3,4 The process of narrowing of cerebral vessels seems to be a reaction of brain blood vessels to a wide variety of external stimuli, injuries, or genetic defects. Conditions such as sickle cell anemia, neurofibromatosis-1, Down's syndrome, congenital heart defects, antiphospholipid syndrome, renal artery stenosis, and thyroiditis have been found to be associated with moyamoya disease in the literature. 5 But more than half of the children seen with this disease have no cause for their moyamoya syndrome. The process of blockage, once it begins, tends to continue despite any known medical management unless treated with surgery. 6 The clinical features of moyamoya disease differ between children and adults. In contrast to adults, who typically present with subarachnoid or intraparenchymal hemorrhage, children with moyamoya disease usually exhibit recurrent transient ischemic attacks (TIA) or infarction in the territory of the ICA, particularly in the frontal lobe. Typical symptoms are monoparesis, hemi paresis, aphasia, and dysarthria. Headache, seizures, and involuntary movements, such as hemichorea, can be 
serious symptoms associated with pediatric moyamoya disease. Some children give history of fall may be due to hyperpnea or excitement, such as playing, blowing on hot noodles, playing a trumpet or crying, may trigger TIA due to hyperventilation-induced reduction of cerebral blood flow. When a precise diagnosis is delayed, slowly progressive mental impairment has also been observed. MRI not only reveals areas of infarctions, but also allows direct visualization of these collateral vessels as multiple small flow voids at the base of brain and basal ganglia. MR angiography is used to confirm the diagnosis and to see the anatomy of the vessels involved. It typically reveals the narrowing and occlusion of proximal cerebral vessels and extensive collateral flow through the perforating vessels demonstrating the classic puff of smoke appearance. ${ }^{7}$ Acute management is mainly symptomatic and directed towards reducing elevated intracranial pressure, improving cerebral blood flow, and controlling seizures. ${ }^{8}$ There are no effective medical therapies for moyamoya disease. Surgical revascularization is thought to improve cerebral perfusion, and to reduce the risk of subsequent stroke in both pediatric and adult patients. In pediatric patients, the incidence of TIA rapidly decreases after surgery. Surgical procedures are classified into three categories, direct bypassing including superficial temporal artery to MCA (STA-MCA) anastomosis, indirect bypassing including encephaloduroarteriosynangiosis (EDAS) and encephalomyosynangiosis (EMS), and combined bypassing. ${ }^{9}$ Direct bypassing canbe technically challenging in some pediatric patients with cortical arteries of smaller diameter, but can improve cerebral hemodynamics immediately after surgery. Indirect bypass surgery that induces spontaneous angiogenesis between the brain surface and the vascularized donor tissues is technically simple, but requires $3-4$ months for the collaterals to develop. ${ }^{10}$ Indirect surgery can provide extensive collaterals in almost all pediatric patients, but only around half of adult patients. Prognosis of patients with moyamoya disease is found to be related to age and the type of presentation. Hemodynamic improvement after surgical procedures appears to be similar in all age groups. TIA and epileptiform clinical pictures have a better long-term outcome when compared to infarctions. ${ }^{11}$ This case highlights that moyamoya disease is an important cause of cerebral stroke in children, early diagnosis and management leads to favorable outcome.

\section{Conclusion}

In conclusion, the diagnosis of moyamoya disease is now accessible to modern brain imaging techniques (MRI and MRA), easily achievable in children. It is important to be familiar with the clinical manifestations and MRI/MRA findings in moyamoya disease or syndrome to make an early diagnosis. The natural course of the disease involves the neurological and vital prognosis in young children, due to repeated ischemic attacks. Their identification would facilitate early diagnosis in order to treat neurosurgically, leading to a better neurological prognosis. 


\section{References}

1. Soriani S, Scarpa P, Voghenzi A. Moyamoya disease in childhood: a familial case report. Child's Nervous System. 1993;9:215-219.

2. Suzuki J, Takaku A. Cerebrovascular "moyamoya” disease. Disease showing abnormal net-like vessels in base of brain. Arch Neurol. 1969;20:288-99.

3. Wetjen N, Garell P, Stence N. Moyamoya disease in the mid western United States. Clinical Neurology and Neurosurgery. 1997;99(2): p. S31- S35.

4. Khan N, Schuknecht B, Boltshauser E. Moyamoya disease and moyamoya syndrome: experience in europe; choice of revascularisation procedures. Acta Neurochirurgica. 2003;145(12): p. 1061-1071.

5. Lutterman J, Scott M, Nass R, and Geva T. Moyamoya syndrome associated with congenital heart disease. Pediatrics. 1998;101(1): p. 57-60.

6. Smith ER, Scott RM. Moyamoya syndrome associated with congenital heart disease. Skull Base. 2005;15(1): p. 15-26.

7. Atlas SW. MR angiography: techniques and clinical applications MRI of the brain and spine. 3rd ed Philadelphia, PA, USA: Lippincott Williams and Wilkins; 2002.

8. Han DH, Kwon OK, Bark BK, Jang B. A cooperative study: clinical characteristics of 334 Korean patients with moyamoya disease treated at neurosurgical institutes (1976-1994). Acta Neurochirurgica. 2000;142(11): p. 1263-1274.

9. Kuroda S, Houkin K. Moyamoya disease: current concepts and future perspectives. Lancet Neurol. 2008;7:1056-66.

10. Houkin K, Nakayama N, Kuroda S, Ishikawa T, and Nonaka T. How does angiogenesis develop in pediatric moyamoya disease after surgery? A prospective study with MR angiography. Childs Nerv Syst. 2004;20:734-41.

11. Inoue TK, Ikezaki K, Sasazuki T, Matsushima T, and Fukui M. Linkage analysis of moyamoya disease on chromosome 6. Journal of Child Neurology. 2000;15(3): p. 179-182. 